\title{
MASTER
}

\section{THEORETICAL HIGH ENERGY PHYSICS}

\author{
- Progress Report \\ for the Period
}

May 1, 1975 - April 30, 1976

$E(11-1)-2271$

1 Principal Investigotors:

T. D, Lee

R. Serber

DI

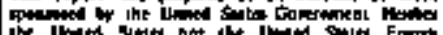

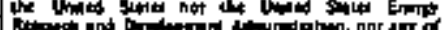

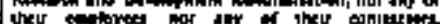

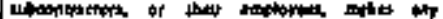

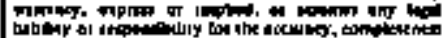

\section{Deportment of Physics \\ Columbia University \\ New York, N. Y. 10027}

Moy 1976

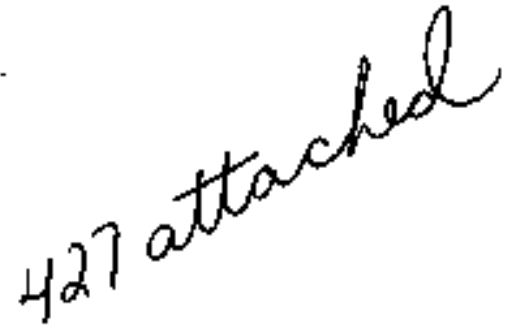

Prepared for

The U. S. Energy Resecrch and Development Administration

under Contract E (11-1)-2271 


\section{DISCLAIMER}

This report was prepared as an account of work sponsored by an agency of the United States Government. Neither the United States Government nor any agency Thereot, nor any of their employees, makes any warranty, express or implied, or assumes any legal liability or responsibility for the accuracy, completeness, or usefulness of any information, apparatus, product, or process disclosed, or represents that its use would not infringe privately owned rights. Reference herein to any specific commercial product, process, or service by trade name, trademark, manufacturer, or otherwise does not necessarily constitute or imply its endorsement, recommendation, or favoring by the United States Government or any agency thereof. The views and opinions of authors expressed herein do not necessarlly state or reflect those of the United States Government or any agency thereof. 


\section{DISCLAIMER}

Portions of this document may be illegible in electronic image products. Images are produced from the best available original document. 


\section{Notice}

This report was prepared as an account of work sponsored by the United States Government. Neither the United States nor the United States Energy Research and Development Administration, nor any of their employees, nor any of their contractors, subcontractors, or their employees, makes any warranty, express or implied, or assumes any legal hobility or responsibility for the accuracy, completeness, or usefulness of any information, apparatus, product or process disclosed or represents that its use would nof infringe privately owned rights. 
Parcentages of Time Devoted to Ptoject by Investigators

Octaber I, 1975 - Oetaber 31, 1976

Name

Professors N. H. Christ

G. Feinbers

H. M, foley

R. Friedberg

T. D. Lee

A. H. Mueller

R. Serber

G. C. Wick

Assistant Professors $M_{1}-Y$, Chen

J. Finkelstein

E. J. Weinberg

Research Associates

C. Bernard

A. Guth

J. Koplik
\% of Time

100

$\sim 22$

$\sim 12$

100

100

100

100

21

100

$\sim 15$

100

100

$\sim 33$

100

50

100

50

100

100

100
Period

3 summer months

$4 \frac{1}{2}$ acodemic months 2 academic months

3 summer months

$1 \frac{1}{2}$ summer months

1 summer month

3 summer months 2 cocodemic months

3 summer months 2 acadernic months

3 summer months

3 summer months

acodentic year

3 summer months academic year

3 summer months acodemic year

2 months

13 months

11 months

Note: During the acodemic year, the average teaching load for our faculty members is one course. A considerable amount of research (in addition to shat specified above) is performed by the severol investigators during thase nine months. 
Progress Report

Professor Norman H. Christ

The majority of Arofessor Christ's research during the past year has been devored to the study of classical and quantum aspects of solitons, especially in gauge theories. In collaboration with a student, Mr. Richord Gonsolves, a detailed theory of the quantum mechanical scattering of two solitons in two-dimensional spoce time has been developed. This method unambiguously defermines the quantum corrections to the semiclassical opproximation to the $S$ matrix. In its present form the procedure is too complex to allow explicit calculation of scattering phase shifts even for the simplest Sine-Gordon theory. However, it is quite likely that considerable simplification is still possible. Mr. Gonsolves' thesis contains a complete account of the work done to date.

Together with $D_{r}$. Alan Guth and Professor Erick Weinberg, Professor Christ hos applied the canonical formalism of Dirae to discuss the symmetry properties of solitan solutions to Yang-Mills theories. It has been discovered that Julia-Zee dyon solutions to the $\mathrm{SU}_{2}$ Higgs model cannot be fransformed into the usual Coulomb gauge, while if the standard axial gauge, $\vec{W}_{3}=0$ is adopted, the behoyior of infinity is so singular that the operations generated by products of angular momenta are not well defined. However, o different (non-hólonomic) choice of boundary conditions in axial gauge ovoids this difficulty and with the use of Dirac's formalism provides a consistent canonical description of operations generated by angular momenta, electromagnetic charge and linear momento which con be applied to Yang-Mills solitan 
solutions. This classical method aflows a simple and direct discussion of charge and angular momentum in the quantum theory of these solitons. A paper describing these results is now being written.

A second student, Mr. Amarendra Sinha, warking with Professor Christ, has been studying, in detail, classical soliton solutions to an $\mathrm{SU}_{3}$ gauge theory containing an actet of Higgs scalar fields. Depending on the nature of the Higgs field minimizing the potentiol three different types of soliton solution ate found. One of these provides an example of a non-abelion Dirac magnetic monopole. In all coses, expressions for the massless field strengths corresponding to the unbroken gauge symmetries have been obtained. A poper describing these results, which differ in a number of interesting respects from the "t hooft-Polyakov $\mathrm{SU}_{2}$ monopole, is currently being wititen.

Finally the asymptotic behovior of the elastic form factors of particles in a gauge theory hove been studied using renormalization group fechniques with a third sfudent, Mr. Ashoke Chatterjee. Upper bounds and leading logorithmic behavior are determined in tems of parameters entering a homogeneous Callon-Symanzik equarion for both abelian and non-abelian vector theories. The conclusions of this investigation appear in Mr. Chatteriee's Ph, O. Thesis and a paper-xurtently being prepared. 
Professot Feinberg has worked principally in two orecs:

a. A calculation was carried ouf, with J. Sucher, of the radiative Ml decoys between singlet and triplef stafes of charmonitm. The rates are found to depend on the details of the quark-quark interaction, and may therefore give important information about these interactions. This work has been published in Physical Review Letters.

b. An altemative model for the psi particles has been developed, with T. D. Lee, In this model, the psi particles are faken to be bound states of a pair of heavy, integer charge fermions, the F-particles. It is possible to fit the known properties of the psi porticles in this model. Also, since the F-porticles are not taken to be confined, they can be produced above threshold, and then decoy weakly, sometimes into charged leptons. These decays may account for the $\mu$-e events absewed in $\mathrm{e}^{+}-\mathrm{e}^{-}$arnithilation by Perl et al. This work hos been submitted to the Physical Review. 
Professor Finkelstein hos worked on three projects:

a) He has studied production of the $\Psi$ particle in photon-induced events, and has at fempted to calculate this cross section in a model which assumes that the $\$$ is composed of chamed quarks. This work has been published in the Physical Review.

b) Together with Joel Koplik, he has studied several problems assaciated with the formulation and use of multiperipheral cluster models and the closely related Reggeon loop exponsion in dual models. They have developed a method to uniquely assign multiparticle events to clusters, and have shown that this assignment leads to equations with Regge-behaved solutions. They have also studied the relation between the pomeron and ordinary Reggeons, and have identified the assumptions needed to obtain frominated pomeron couplings. This work hos been reported as preprint CO-227I-72.

c) Professor Finkelstein has also been studying the energy dependence of the "effective trajectory" which can be exchanged in non-diffractive high-energy scattering. Even in models which predict a pure power behavior with energy of non-diffractive cross sections at sufficiently large energy, it is possible that there may be deviations from this simple behavior at lower energy. He hos studied the relationship between these deviations and the observed non-constancy of total cross sections. A report of this work is in preparation. 
Professor Foley has been involved with two projects during the past year:

a) The explanation of the sign reversal of the alkali atom excited states for $\ell>1$ was published with R. Sternheimer of Brookhaven in Physics Letters. Dr. Stemheimer is continuing this study for a number of such states, including the case $Q=1$ (where the effects of core exchange polarization perturb the fine structure but do not reverse it). Professor Foley may join him in some of this work, but more likely he will be interested in the more general problem of the refation of excitations to the asymptotic wave function.

b) The project on cascade mechanisms in exotic atoms, $\bar{p} \overline{\mathrm{H}}, \bar{p} \bar{\pi}, \mathrm{p} \overline{\mathrm{K}}, \mathrm{PF}$, efc., with Professor Foley's student, David Chang, has gone slowly but is essentially finished. The inetastic process, in which energy is transferred to heavy-particle motion of the coiliding atoms is shown to be significant, indeed dominating, for highly excited states with the heavier negative particles. The dependence of the cascade rate on particle mass and energy has been worked out. 'Heating' effects are importont in some cases in determining final distributions. 
During this past year, Professor Friedberg hos worked with Professors Lee and Sirlin on a specific model of a "nontopologicai" soliton in three dimensions. The model chosen involved two scalar fields, one of them complex, and was the simplest locat field theory that would be renormolizable in quantum theory and would give rise to "soliron" solutions. These solutions are not time-independent but ore steadily rotating at angular velocity $\omega$ in the complex plane of the charged field. They are characterized by a chorge $Q$ (the "momentumu" conjugate to $w$ ) and an energy $E$. Friedberg, Lee and Sillin have investigated the relation between $E$ and $Q$ analyt+ ically for extreme values of $\omega$ and numericalfy for intermediate values. They ex amined the quantization of small deviations from these solutions by the Christ-Lee method. They established that for all $Q$ the lowest-E soliton solution is stable against small perturbations, and for sufficiently large $Q$, stoble as well againsł large perturbarions (i,e., ogainst decay into free mesons).

With his student, P. Gujrati, Professor Friedberg has worked on diagrommatic highfemperature expansion of the partition function of the spin- $\frac{1}{2}$ isotropic Heisenberg model. This work is near completion. 
Progress Report

Dr. Alan H. Guth

During the past year Dr. Guth has been working on two separate projects. The first is being carried out in coltaborotion with Marvin L Goldberger and Dovison E. Soper, both of Princton University. Motivated by the quark model, they are examining the predictions of quantum field theory for the high energy behovior of processes involving bound states. In the past year they have obtained results on three closely rejated problems. First, they hove analyzed in a specific model the behovior of the Bethe-Salperer wave function for a iwo-particle bound state in the limit that the four-momenturn-squared of one leg becomes infinite while the other leg is fixed. (The model contains two spin- $-\frac{1}{2}$ quarks bound by the exchange of o scalar gluan in ladder approximation.) Second, they have studied the conformal invariant operator product expansion of two spinor fields, and have derived explicit expressions for those terms which contribute to the wave function of the pion. This exponsion governs certain asymptotic limits of the true wove function. Finally, they have put this information together to derive the lorge $q^{2}$ behavior of the electromagnetic form factor of the pion. A preprint has been prepared.

The second project involves a collaboration with Norman $\mathrm{H}$. Christ and Erick J. Weinberg. They are studying various properties of the magnetic monopole solutions discovered by G. 't Hooft. In particular, they have developed a elassical canonical formalism in which the 't Hooft solution and alf reasonable modifications of it are regular (nonsingular). The regularity is achieved by working in t',e axial gauge. The canonical formalism in this gange is found to involve subtleties which require special treat- 
ment. Because of the absence of singulorities, thef fllopose that the axiol gauge provides an unambiguous formalism for studying bull the clossical and semi-classical properties of these monopole solutions. 
Dr. Koplik has been working on the following two projects:

a) High transverse momentum scattering off nuclei: An atfempt was mode to understand the unexpected enhancement observed in high energy hadron inclusive cross sections at high transverse momentum off nuclear targets. The existing data and the known properties of such reactions with hadron torgets were seen to rule out the most obvious explanation-incoherent multiple scattering. Unfortunately, the limited available information does not allow an understanding of the phenomenon without further, ad hoc, assumptions.

b) Dual topological expansions (with J. Finkelstein): They have studied several aspects of the so-calted dual topological expansion, wherein one aftempts to incorporate the Regge and duality phenominology of hadrons into a classification of scattering amplitudes occording to topological structure. They hove examined in detail the consequences of various final state counting schemes, approaches to the Reggeon bootstrap, the relation between the pomeron and other Reggeons, and the possible absence of Regge cuts. 
Progress Report

Professor T. D. Lee

During this past year Professor Lee has worked in the following areas:

1. Soititons

A systematic survey hos been made to search for all soliton solutions that exist in relativistic local field theories, on both the classical and the quantum levels. Classically o soliton solution is defined to be one that is confined in space at all time. For a renormalizable theory, it can be shown that [N. H. Christ and T. D. Lee, Phys. Rev. 012, 1606 (1975) ] for every classical soliton solution, there exists a corresponding quantum soliton solution. When the nonlinear coupling $g \rightarrow 0$, both the moss and the form factor of the quantum solitan go over to their respective classical limits.

Most of the soliton solutions are in one space dimension. Recently [T.D.Lee,
[ Physics Reports 23C, 254 (1976)], a new class of three space-dimensional soliton solutions hos been found. Its physical structure is studied and the derailed quantization procedure is cartied out [Friedberg, Lee and Sirlin, Phys. Rev.D, to be published)]. Some general mathematical theorems on stability problems of such nonlinear solvitions are estab! ished; some of them are quite new, apporently unknown to mathematicions working on partial differential equations. Generalizations to gange theories and to include spin $\frac{1}{2}$ fermions are being carried out at present.

The relation between the soliton solution and the ustual boundstate solution in terms of the Bethe-Salpeter equation has also been analyzed recently [preprint CO-2271-76]. This leads to a new insight into the boundstate problem in quantum mechanics. Usually, it is thought that in order to hove stable boundstotes, quantum 
meghanics must be crucial. This turns out to be not the case. As shown in the aforemenfioned papers, the solitons are boundstates that already exist on o classical level. Furthermore, when the nonlinear coupling $\mathrm{g}$ is small, the quantum solution alwoys approaches its classical limit. For a multi-body system, the classical description is, of course, much simpler than the corresponding quantum description.

\section{Psions}

In a retent paper [Feinberg and Lee, CO-22.71-74], the psions are identified as boundstates of a new heavy spin $\frac{1}{2}$ fermion $F$ and its antiparticle $\bar{F}$. In orcier to account for the various observed decays of $\psi(3.7)+J / \mu(3.1)$, the $F$ particles are forsin to have a small coupling $f_{-}$to $C=-1$ mesons, but they must have a relatively lorgo coupling $f_{+}$to $C=+1$ mesons. Because of the narrow width of $J / 4(3,3) \rightarrow$ hadrons and $\psi(3.7) \rightarrow$ hodrons, the coupling $(4 \pi)^{-1} f_{-}^{2}$ is $\approx 10^{-3}$. From the observed widths of

and

$$
\begin{aligned}
& \psi(3.7) \rightarrow J / \psi(3.1)+2 \pi \\
& \psi(3.7) \rightarrow J / \psi(3.1)+\eta
\end{aligned}
$$

and the fact that both reactions have very small phase space and matrix elements that represent forbidden transitions, the large coupling $f_{+}^{2} / 4 \pi$ is found to be $\approx 1$. This analysis shows that the F's have very weak interactions with vector mesons, but strong interactions with spin 0 mesons, in contrast with the usual hadrons. Thus, it throws daubi on the conventional idea that psions are mode of chormed quarks, satisfying approximate $\mathrm{SU}_{4}$ symmeiry.

The same model is then used to explain the continum contribution to $\mathrm{e}^{+} \mathrm{e}^{-}-$ 
hadrons at SPEAR above $4 \mathrm{GeV}$. Other consequences of dilepton events from $\mathrm{e}^{+} \mathrm{e}^{-}$, neutrinos and p-p collisions are also discussed.

\section{Abnormal Nucleor States}

Work connected with the possible existence of a new form of matter at high density suggested by Lee and Wick [Phys. Rev. D9, 2291 (1974)] is being continued. One of the questions is what is the best experimental direction in which to investigate such problems. In this connection the article by T. D. Lee [in LBL Proposal-32, prepared for ERDA, May T975] points to the importance of constructing a third heary ion injector for the Super-HILAC and a high vacuum liner for the Bevalac Heavy Ion Facility at the Lowrence Berkeley Laboratory. It is hoped that the proposed construction will extend the copability of the Bevaloc to produce relativistic ions up to uranium, and increase its versatility to produce ions in the intermedicte 35 to $200 \mathrm{MeV} /$ amu range; thereby it may open the doot to studying possible new coherent nuclear states at twice the normal nuclear density.

4. Besides the above-mentioned topics, Professor Lee is continuing his work on CP nonconservation and spontaneous symmetry breaking, quesłions related to vacuum excitation and vacuum stability, the possible existence of the intermediate boson, further applications of field algebra, high energy neutrino reactions, scaling properties, and, above all, a possible unification of the electromagnetic, weak and strong interactions. 
Professor Mueller hos worked on two topics;

i) He and Joel Koplik have finished the work which was in progress at this time lost year on nuclear collisions (published in Physics Letters and Physical Review). Theit now rather firm conclusion is that no standard model of high-energy collisions (except possibly o reformulated string model) seems copable of coming close to the experimental data presently available. Defailed predictions and constroints are given for soft field theory and string models of high-energy hadronmuclevs coltisions.

ii) The possibility of the string model's satisfying the constraints imposed by hadronnucleus collisions hos motivared Professor Muefler to make a detailed study of the space-time motions of the relativistic string as it passes through hadronic matrer. At present he is trying to construct a modified string model which ovoids the disostrous zero point fluctuations which are presently in the string model. 
An account of the nuclear model described in tast year's report has been published in the proceedings of the Los Alamos Conference "High Energy Physìcs and Nuclear Structure". An even simpler model has been considered in which the forces are only a Yukawa potential and a repul sive core. This model gave fairly good agreement with the charge densities measured for heavy nuelei. A paper on this subject has been written and submitted to Physical Review. 
Progress Report

Assistant Professor Erick Weinberg

During the past year, Professor Weinberg's general field of interest has continued to be the use of semi-classical appraximations in field theory. Recently there hos. been hope that these methods might provide a new way of abtaining composite stotes in quantum field theory and might uttimately be useful in obtaining a realistic description of the hadrons.

Most of Professor Weinberg's work has been on the magnetic monopole solution of 't Hooft; the work has been in collaboration with Norman Christ and Alan Guth. Their infent was to apply the semi-classical method of Christ and Lee to the "t Hooft solution, in order to deternine the spectrum of states. They found in addifion to the monopole, a series of electrically charged monopoles, with quantized electric charge, which are almost degenerate with the monopole; previously Julia and Zee had found the clossical solutions corresponding to these states, but had not been able to provide a mechanism for quantizing their charge. In the course of their investigations, they were led to consider several Yang-Mills gauges; in particular, they found that the axial gauge possesses subtleties not previously noticed, If these are not treated properly, there are difficulties in the definition of the generators of symmetry transfomations, e.g., a naive treatment lereds to angular momentum operators which do nof have the rotation group commutation relations. They have developed a method, using the method of Dirac brockets, to handle these difficulties. A poper dealing with this work and with their work on the ' $\mathrm{H}$ Hooft monopole is in preparation. 
Professor Weinberg has also, in collaboration with A. Guth, begun to consider monopole configurations with magnefic charge greater than one. Their work is still preliminary, but there are indications that these may possess some rather unstual properties. 
Professor Wick's attempt to opply the Green's function method to soliton problems was abandoned, at least temporarily. Some simple results obtained with it were published independently by Jackiw and Goidstone. Professor Wick's hope was reatly to go further and study, for instance, seattering problems, but questions of non-commutativity proved quite intractable.

Professor Wick's next effort was in the direction of irying to connect the method developed by Rofessors Christ and Lee with the cononical tronsformation results of Faddeev. There are some really interesting questions here. He was able to show thaf the "coordinate transformation" of Christ-Lee can be exiended in a natural way to a canonical transformation, at least in the neighborhood of a "soliton solution". As is well known, linear canonical tronsformutions can be transferred from classical to quantum mechanies without serious difficulty, and Professor Wick is fairly convinced that it should be possible to apply the formalism he has developed to quantum effects. The purely classical part of this work was described in a brief report at the Paris meeting of last June; if he con see clearly the application to quantum field theory, the work may be published.

Another study in a connected context hos been the development of a theorem on the unitary operator corresponding to the canoni cal transformation generated by linear equations of motion. This is a quite general result, opplying olso when the coefficients of the equations are time-dependent and the number of degrees of freedom is arbitraty. 
As far as Professor Wick can tell, the result is new, which seems rother susprising.

Finally, he hos been interested in "fopological stability" of certain classes of solitons. This has led, so far, only to a better understonding of the work of ' $t$ Hooft and Mandelstam on monopole solutions. 


\section{PUBEICATION LIST}

$\varepsilon(11-1)-227$

1. A. Chatterijee ond N. H. Christ, "Asymprotic Behavior of Form Factors in Vector Field Theories" (to be submitted to Physical Review).

2. N. H. Christ and T. D. Lee, "Quontum Expansion of Soliton Solutions", Phys. Rev. D12, 1606 (1975).

3. N. H. Christ, "Quantum Exponsion of Soliton Solutions", of the Conference "Extended Systems in Field Theory", Phys, Reports 23C, 294 (1976).

4. N. H. Christ, A. H. Guth and E. J. Weinberg, "Canonical Fomalism for Gauge Theories with Application to Monopoles" (to be submitted to Phys. Rev. D).

5. G. Feinberg, "Decays of \& Mesons", Phys, Rev, D12, 109 (1975).

6. G. Feinberg, "Neutral Current Phenomena", in High Energy Physics and Nuclear Structure - 1975 (AlP Conference Proceedings No. 26), p. 468.

7. G. Feinberg, "Polarized Electron-Nucleus Scattering and Parity-Viplating NeutralCurrent Interactions", Phys, Rev, D12, 3575 (1975).

8. G. Feinberg and J. Sucher, "Relativistic Colculation of Radiative MI Transitions in Charmoni um", Phys. Rev. Letr. 35, 1740 (1975).

9. G. Feinberg and 5 . D. Lee, "Unconfined, Heavy Fermion Model of $J / \psi$ Particles" (to be pubfished in Phys. Rev. D).

10. J. Finkelstein, "An Analysis of the $\psi \mathrm{p}$ Tofal Cross Section", Phys. Rev, D!1, 3337 (1975).

11. J. Finkelstein and J. Kaplik, "f-Dominance and Final-State Counting in Multiperipherical Cluster Models" (to be published in Phys. Rev, D).

12. J. Finketstein and 1. Koplik, "The Energy Dependence of the p Regge Trojectory" (to be submitied to Phys, Rev. D).

13. H. M. Foley and R M Stemheimier, "Fine-Structure Inversion of the Third Exci ked State of Sodium", Physics Letters 55A, 276 (1975).

14. R. Friedberg and J. M. Luttinger, "Density of Electronic Energy Levels in Disordered Systems", Phys. Rev. 812, 4460 (1975). 
15. R. Friedtherg, T. D. Lee and A. Sirlin, "A Class of Seaior-field Soliton Solutions in There Space Dimensions" (to be published in Phys.Rev. D).

16. R. Friedberg and B. Coffey, "Singie-Mode Superfluorescence Theory Compared with Experiment", Phys. Rev, Al3, 1645 (1976).

17. A. Guth, M Goldberger and D. Soper, "The Large $Q^{2}$ Behavior of the Pion Electromognetic Form Foctor" (to be published in Phys. Rev.)

18. A. Guth and D. Soper, "Short-Distance Behavior of the Bethe-Salpeter Wove Function", Phys.Rev. D12, 1149 (1975).

19. J. Koplik and G. Chu, "Multiperipheral Model of Direct Muan Production", Phys, Rev. 비, 3134 (1975).

20. J. Koplik and G. Chu, "Direct Muon Production in a Multiperipheral Model", Phys, Lett. 55B, 466 (1975).

21. J. Koplik and J. Dash, "Energy Scales and Diffroction Scattering", Phys, Rev, D12, 785 (1975).

22. J. Koplik and A. H. Mueller, "On the Abromovskii, Kancheli, Gribov Reggeon Cutting Rules", Phys. Lett. 58B, 166 (1975).

23. J. Koplik and A. H. Mueller, "Hadron-Nucleus Collisions at High Energies", Phys. Rev. D12, 3638 (1975).

24. T. D. Lee, "A Possible New Form of Matter at High Density", in Report of the Workshop on 8EV/Nucleon Collisions of Heavy lons (BNL, 1975), p. 1.

25. T. D. Lee and M. Morgulies, "Interaction of a Dense Fermion Medium with a Scalar Meson Field", Phys, Rev. D11, 159 (1975).

26. T. D. Lee, "Examples of Four-Dimensional Soliton Salutions and Abnormal Nuclear States", at the Conference "Extended Systems in Fie!d Theory", Phys. Reports 23C, 254 (1976).

27. T. D. Lee, "Exomples of Nontopológical Soliton Solutions" (to be published).

28. M. Margulies, "Multiloop Colcutotions in the $\sigma$-Model", Phys. Rev, D13, 1621 (1976).

29. M. Margulies, "Abnormal Nuclear States: Higher Order Corrections to the SemiClassical Solution", Phys, Rev, D13, 1642 (1976). 
30. A P. Sothe, "W boson Pairs: Their Photoproduction and Decay to Leptons", Phys, Rev. D11, 1940 (1975).

31. A, Sen, "Confinement and Shielding in a Massive-Massless Schwinger Model" (to be published in Phys, Rev.).

32. A. Sen, "On a Simplified Polyakor Model" (to be published in Phys. Rev.).

33. R. Serber, "A Simple Nuclear Modiel", in High Energy Physics and Nuclear Structure - 1975 (AIP Conference Proceedings No. 26), P. 700.

34. R. Serber, "A Simple Nuclear Model" (ro be published in Phys. Rev. C).

35. E. J. Weinberg and A. H. Guth, "Non-existence of Spherically Symmetric Monopoles with Multiple Magnetic Charge" (to be submirłed to Phys. Rev.)

36. W. Yueh, "Comments on Vacuum Polarizations and Non-classical Monopole Theory", Phys. Rev, D12, 3221 (1975).

\section{DISSERTATIONS}

A. Chatterjee, "Asysnprotic Behavior of Form Factors in Vector Field Theories", 1976.

R. Gonsolves, "On the Quantization of Soliton Scattering Solutions in Model Field Theories", 1976.

M. Margulies, "Contribution to the Study of Abnomal Nuclear States and Relatec.

Problems: A, Interaction of a Dense Fermion Mediurn with a Scalar-Meson Field

B. Multiloop Calculations in the o -Model

C. Abnomal Nuclear Stotes: Higher Order Corrections to the Semiclassical Solution", 1976.

A. Sen, "On a Simple Polyakov Modef", 1975. 